\title{
GEOMETROTHERMODYNAMICS OF BLACK HOLES IN LORENTZ NON-INVARIANT MASSIVE GRAVITY
}

\author{
Alberto Sánchez* \\ Departamento de Posgrado, CIIDET, \\ AP752, Querétaro, QRO 76000, MEXICO
}

(Dated: June 8, 2022)

\begin{abstract}
We analyze a static and spherically symmetric hairy black hole solution in non-invariant massive gravity. The formalism of geometrothermodynamics is used to describe the thermodynamic characteristics of this black hole in a Legendre invariant way. For a black hole in massive gravity, the geometry of the space of equilibrium states is computed showing that it contains information about the thermodynamic interaction, critical points and phase transitions structure.
\end{abstract}

Keywords: Thermodynamics, Phase transitions, Black Holes

PACS numbers: 05.70.Ce; 05.70.Fh; 04.70.-s; 04.20.-q

*Electronic address: asanchez@ciidet.edu.mx 


\section{INTRODUCTION}

The search for a quantum theory of gravity has received attention of many researchers. These investigations have suggested that general relativity will be superseded by a quantum theory of gravity at high enough energies with respect to the Planck mass or the corresponding Planck length [1]. The main idea is that general relativity is valid as an effective field theory for length scales much larger that Planck length. If this theory is true, the possibility to proof it by experiments is almost hopeless. On the other hand, the most successful cosmological model that is in agreement with the observational data implies the existence of a vacuum energy related with the cosmological constant $\Lambda$, whose magnitude is unnatural from the effective field theory point of view [2]. Hence, a dark energy is needed to reconcile general relativity with the observations. The dark energy solves the problem of the accelerated expansion of the Universe. Nevertheless, the interest to explain the acceleration of the Universe without resorting to the dark energy has motivated the research for large-distances modified theories of the gravity; the Lorentz-breaking massive gravity is one of these models which is free from pathologies such as ghosts, low strong coupling scales or instabilities at full non-perturbative level [3 $[5]$. Although these models do not require the existence of $\Lambda$, the cosmological constant problem remains open. A generalized Schwarzschild solution for this model has been obtained by D. Comelli et al. which is an exact black hole solution showing a nonanalytic hair [6].

The study of the thermodynamics of black holes and its relationship with geometry has been a subject of intensive research [7-12]. This geometric study has been considered in

several papers by means of different approaches like Weinhold's theory [13], Ruppeiner's theory [14] or the most recent theory called geometrothermodynamics [15]. Geometrothermodynamics (GTD) is a formalism that relates a contact structure of the phase space $\mathcal{T}$ with the metric structure on a special subspace of $\mathcal{T}$ called the space of equilibrium states $\mathcal{E}$. In this work, we will use a Legendre invariant metric in the context of geometrotermodynamics to formulate an invariant geometric representation of the thermodynamics of a static and spherically symmetric hairy black hole solution in massive gravity.

This paper is organized as follows. In Section II, we review the most important aspects of the generalized Schwarzschild solution for massive gravity, emphasizing the thermodynamic interpretation of its physical parameters. In Sec. III, we review the fundamentals of GTD. 
In Sec. IV, we investigate the GTD of a black hole in massive gravity and show that it agrees with the results following from the analysis of the corresponding thermodynamic variables. Finally, in Sec. V, we present the conclusions of our work.

\section{LORENTZ-BREAKING MASSIVE GRAVITY}

The general action that describes massive gravity is given by the expression [6, 16 20],

$$
s=\int_{\mathcal{M}} d^{4} \sqrt{g}\left[-\frac{1}{16 \pi} R+\Lambda^{4} \mathcal{F}\left(X, V i, W^{i j}\right)\right]-\int_{\partial \mathcal{M}} d^{3} \sqrt{\gamma} \frac{K}{8 \pi},
$$

where $\mathcal{F}$ is a function of four scalar fields $\phi^{\mu}$ that are minimally coupled to gravity and

$$
X=\Lambda^{-4} g^{\mu \nu} \partial_{\mu} \phi^{0} \partial_{\nu} \phi^{0} ; V^{i}=\Lambda^{-4} \partial^{\mu} \phi^{i} \partial_{\mu} \phi^{0} ; W^{i j}=\Lambda^{-4} \partial^{\mu} \phi^{i} \partial_{\mu} \phi^{j}-\frac{V^{i} V^{j}}{X}
$$

Spacial and spacetime indices are denoted by latin and greek letters, respectively. The second integral is the Gibbons-Hawking-York boundary term [21, 22], where $\gamma$ is the metric induced on the boundary $\partial \mathcal{M}$ and $K$ is the trace of the extrinsic curvature $K_{i j}=\frac{1}{2} \gamma^{k}{ }_{i} \nabla_{k} n_{j}$ of $\mathcal{M}$ with unit normal $n^{i}$. Such a boundary term is required to have a well-defined variational principle in the presence of the border $\partial \mathcal{M}$.

Spherically symmetric black holes in massive gravity have been investigated in [6, 21 23] and it was found that a set of coordinates always can be found where the solution can be written in the form

$$
d s^{2}=f(r) d t^{2}+f(r)^{-1} d r^{2}+r^{2}\left(d \theta^{2} \sin ^{2} \theta d \varphi^{2}\right)
$$

and the fields are given by the expressions [21]:

$$
\phi^{0}=\Lambda^{2}[t+h(r)], \quad ; \quad \phi^{i}=\phi(r) \frac{\Lambda^{2} x^{i}}{r}
$$

The metric functions $f, h$ and $\phi$ are given by the following equations, 


$$
\begin{aligned}
& f=1-\frac{2 M}{r}-\frac{Q}{r^{\lambda}} \\
& h= \pm \int \frac{d r}{f}\left[1-f\left(\frac{\lambda(\lambda-1) Q}{12 m^{2} r^{\lambda+2}}+1\right)^{-1}\right]^{\frac{1}{2}}, \\
& \phi=r,
\end{aligned}
$$

where $M$ and $Q$ are integration constants and $\lambda \neq 1$ is a positive constant. In the case $\lambda>1$ the gravitational potential is asymptotically Newtonian and the parameter $M$ coincides with the ADM mass, while $Q$ is a scalar charge whose presence reflects the modification of the gravitational interaction as compared to General Relativity.

The roots of the lapse function $f\left(g_{t t}=0\right)$ define the horizons $r=r_{ \pm}$of the spacetime. In particular, the null hypersurface $r=r_{+}$can be shown to correspond to an event horizon, which in this case is also a Killing horizon, whereas the inner horizon at $r_{-}$is a Cauchy horizon. Therefore from $f\left(r_{+}\right)=0$ [19] we get,

$$
1-\frac{2 M}{r_{+}}-\frac{Q}{r_{+}^{\lambda}}=0
$$

From the expression (8) for the horizon radii we obtain the following relationship,

$$
M(S, Q)=\frac{1}{2} r_{+}\left[1-\frac{Q}{r_{+}^{\lambda}}\right],
$$

From the area-entropy relationship, $S=\pi r_{+}^{2}$, the equation (8) can be rewritten as

$$
M(S, Q)=\frac{1}{2}\left(\frac{S}{\pi}\right)^{\frac{1}{2}}\left[1-\frac{Q}{\left(\frac{S}{\pi}\right)^{\frac{\lambda}{2}}}\right] .
$$

This equation relates all the thermodynamic variables entering the black hole metric in the form of a fundamental thermodynamic equation $M=M(S, Q)$. Equation (10) is an inhomogeneous function in the extensive variables $S$ and $Q$. Following Davies [24], we homogenisize the fundamental equation by redefining the parameter $Q$ as,

$$
Q=q^{\frac{\lambda}{2}}
$$


Then, equation (10) becomes a homogeneous function of degree $\frac{1}{2}$ in the extensive variables. This procedure was performed explicitly in the context of GTD in [25]. In this case Euler's theorem takes the form [24],

$$
\frac{1}{2} M=T S+\Theta q^{\frac{\lambda}{2}}
$$

with

$$
\Theta=\frac{2 \Phi q}{\lambda q^{\frac{\lambda}{2}}}=\frac{2 \Phi}{\lambda} q^{1-\frac{\lambda}{2}}
$$

Differentiating equation (12) and using the first law of thermodynamics we get,

$$
\frac{1}{2} d M=d M-\Phi d Q+S d T+\frac{\lambda}{2} \Theta q^{\frac{\lambda}{2}-1} d Q+q^{\frac{\lambda}{2}} d \Theta .
$$

Now, we use (13) in order to obtain,

$$
d M=-2 S d T-2 q^{\frac{\lambda}{2}} d \Theta .
$$

The last relation implies the following thermodynamic equilibrium conditions,

$$
\begin{aligned}
S & =-\frac{1}{2} \frac{\partial M}{\partial T}, \\
q^{\frac{\lambda}{2}} & =-\frac{1}{2} \frac{\partial M}{\partial \Theta} .
\end{aligned}
$$

According to the first law of the thermodynamics, the expression for the temperature $T$ and the potential $\Theta$ are given by the thermodynamic equilibrium conditions: $T=\left(\frac{\partial M}{\partial S}\right)_{q}$ and $\Theta=\left(\frac{\partial M}{\partial q}\right)_{S}$, which lead to the following results,

$$
\begin{aligned}
& T=\frac{1}{4 \pi} \frac{\left(\frac{S}{\pi}\right)^{\frac{\lambda}{2}}-q^{\frac{\lambda}{2}}(1-\lambda)}{\left(\frac{S}{\pi}\right)^{\frac{1+\lambda}{2}}} \\
& \Theta=-\frac{\lambda}{4}\left(\frac{S}{\pi}\right)^{\frac{1-\lambda}{2}} q^{\frac{\lambda}{2}-1} .
\end{aligned}
$$


It is easy to show that the temperature (18) coincides with the Hawking temperature. The temperature $T$ will be positive when $\left(\frac{S}{\pi}\right)^{\frac{\lambda}{2}}-q^{\frac{\lambda}{2}}(1-\lambda)>0$, i.e., if $\left(\frac{S}{\pi}\right)^{\frac{\lambda}{2}}>q^{\frac{\lambda}{2}}(1-\lambda)$. The temperature increases rapidly as a function of entropy $S$ until it reaches its maximum value at $\left(\frac{S}{\pi}\right)^{\frac{\lambda}{2}}=q^{\frac{\lambda}{2}}\left(1-\lambda^{2}\right)$. Then, as the entropy increases, the temperature becomes a monotonically decreasing function. The behavior is shown in fig.1

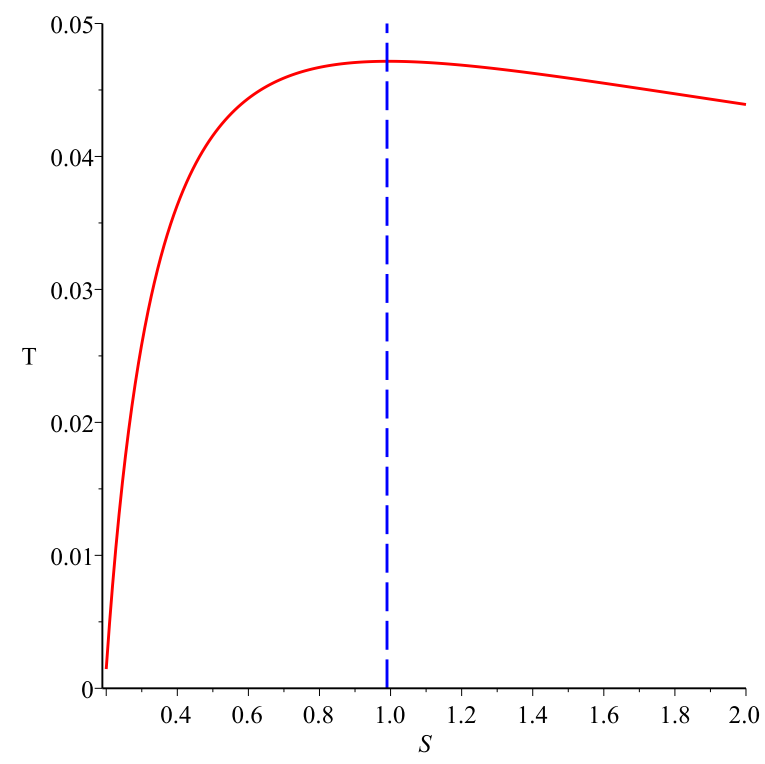

FIG. 1: The temperature $T$ as a function of the entropy $S$, with $\lambda=\frac{1}{2}$ and $q=1$.

The heat capacity at constant values of $q$ is given as

$$
C_{q}=T\left(\frac{\partial S}{\partial T}\right)_{q}=\left(\frac{\frac{\partial M}{\partial S}}{\frac{\partial^{2} M}{\partial S^{2}}}\right)_{q}
$$

where the subscript indicates that derivatives are calculated keeping the charge constant. Using the fundamental equation (10) we get,

$$
C_{q}=-\frac{2 S\left[\left(\frac{S}{\pi}\right)^{\frac{\lambda}{2}}+q^{\frac{\lambda}{2}}(\lambda-1)\right]}{\left[\left(\frac{S}{\pi}\right)^{\frac{\lambda}{2}}-q^{\frac{\lambda}{2}}\left(1-\lambda^{2}\right)\right]}
$$

According to Davies [24], second order phase transitions take place at those points where the heat capacity diverges, i. e., for 


$$
\left(\frac{S}{\pi}\right)^{\frac{\lambda}{2}}-q^{\frac{\lambda}{2}}\left(1-\lambda^{2}\right)=0
$$

these points exist in the interval $1-\lambda^{2}>0$. This behavior is depicted in figure 2 .
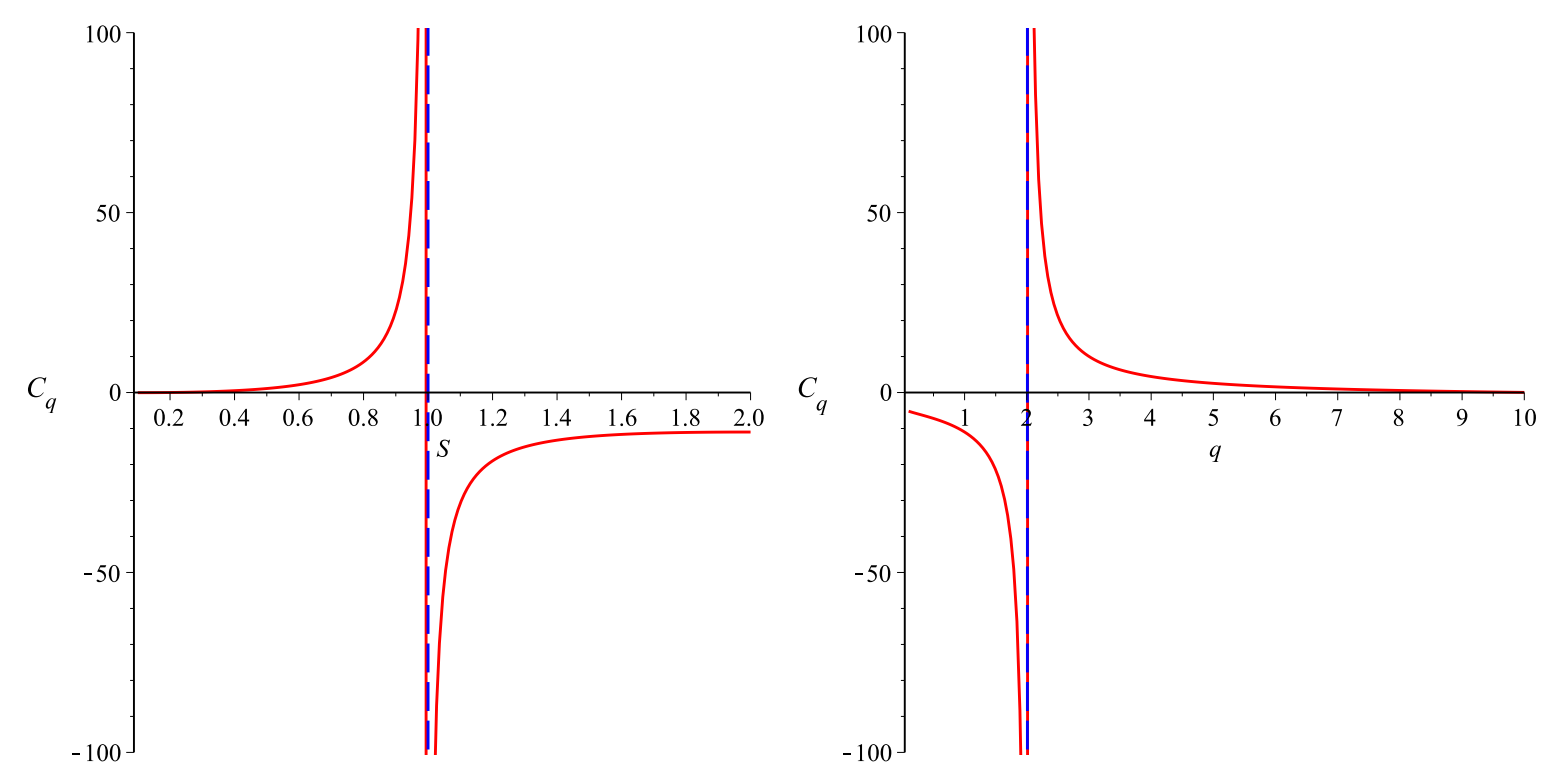

FIG. 2: The heat capacity $C_{q}$ as a function of the entropy $S$ (left) with $q=1$ and as a function of the charge $q$ (right) with $S=2$. In both case we have chosen $\lambda=\frac{1}{2}$,

In the physical region with $\left(\frac{S}{\pi}\right)^{\frac{\lambda}{2}}-q^{\frac{\lambda}{2}}(1-\lambda)>0$. i.e., the region with positive temperature, the heat capacity is positive in the interval,

$$
q^{\frac{\lambda}{2}}(1-\lambda)<\left(\frac{S}{\pi}\right)^{\frac{\lambda}{2}}<q^{\frac{\lambda}{2}}\left(1-\lambda^{2}\right)
$$

indicating that the black hole is stable in this region. At the maximum value of the temperature which occurs at $\left(\frac{S}{\pi}\right)^{\frac{\lambda}{2}}-q^{\frac{\lambda}{2}}\left(1-\lambda^{2}\right)$, the heat capacity diverges and changes spontaneously its sign from positive to negative. This indicates the presence at second order phase transition which is accompanied by a transition into a region of instability.

\section{REVIEW OF GEOMETROTHERMODYNAMICS}

Geometrothermodynamics is a theory that has been formulated in order to introduce the Lagendre invariance in the geometric description of the thermodynamic equilibrium states 
[15]. This theory has been applied to different thermodynamic systems like black holes, ideal gas or Van der Waals gas [26, 27, 30 35]. In all the cases investigated so far, GTD leads to consistent results that describe geometrically the phases transitions and thermodynamic interaction using Legendre invariant metrics.

The main ingredient of GTD is a $(2 n+1)$-dimensional manifold $\mathcal{T}$ with a set of coordinates $Z^{A}$ which allow us to define a non-degenerate Legendre invariant metric $G$ together with a linear differential 1-form $\Theta$ which fulfills the condition $\Theta \wedge(d \Theta)^{n} \neq 0$, where $n$ is the number of thermodynamic degrees of freedom, $\wedge$ represents the exterior product and $d$ the exterior derivative. In GTD, we also have the space of thermodynamic equilibrium states which is a submanifold $\mathcal{E} \subset \mathcal{T}$ defined by means of a smooth embedding mapping $\varphi: \mathcal{E} \longrightarrow \mathcal{T}$ such that the pullback $\varphi^{*}(\Theta)=0$.

With the above elements a metric $g$ is induced in $\mathcal{E}$ by means of $\varphi^{*}(G)=g$, giving a Riemannian structure to this space. So, in GTD the physical properties of a thermodynamic system in a state of equilibrium are described in terms of the geometric properties of the corresponding space $\mathcal{E}$.

The set of elements $(\mathcal{T}, \Theta, G)$ with the conditions above mentioned is called a contact Riemannian manifold. If we consider the $2 n+1$-dimensional space $\mathcal{T}$ coordinatized by the set $Z^{A}=\left\{\Phi, E^{a}, I^{a}\right\}$ where $A=0, \ldots, 2 n$ and $a=1, \ldots n$, the 1 -form $\Theta$ will be

$$
\Theta=d \Phi-I_{a} d E^{a}
$$

We choose now the subset $E^{a}$ as coordinates of $\mathcal{E}$. Then, the mapping $\varphi$ is given by

$$
\varphi:\left(E^{a}\right) \longrightarrow\left(\Phi, E^{a}, I^{a}\right)
$$

and the condition,

$$
\varphi^{*}(\Theta)=\varphi^{*}\left(d \Phi-\delta_{a b} I^{a} d E^{b}\right)=0,
$$

leads to the standard conditions of the thermodynamic equilibrium and the first law of thermodynamics, 


$$
\frac{\partial \Phi}{\partial E^{a}}=I_{a}, \quad d \Phi=I_{a} d E^{a}
$$

The second law of thermodynamics under this formalism is written as,

$$
\frac{\partial^{2} U}{\partial E^{a} \partial E^{b}} \geq 0, \quad ; \quad \frac{\partial^{2} S}{\partial F^{a} \partial F^{b}} \leq 0
$$

where $U$ and $S$ represent the energy and entropy for each of the corresponding thermodynamic systems. Here $E^{a}\left(F^{a}\right)$ represent all the extensive variables other than $U(S)$.

In GTD the only requirement for defining a metric $G$ of the space $\mathcal{T}$ is that it fulfills the condition of Legendre invariance; therefore, we have many possibilities of constructing a metric with these features. In fact, all the Legendre invariant metrics found so far can be classified in three classes. It turns out that each class can be used to describe thermodynamic systems with particular phase transitions [27]. For instance, for systems with second order phase transitions, the most general metric can be expressed as,

$$
G=\Theta^{2}+\left(\delta_{a b} E^{a} I^{b}\right)\left(\eta_{c d} d E^{c} d I^{d}\right)
$$

where $\delta_{a b}=\operatorname{diag}(1,1, \ldots, 1)$ and $\eta_{a b}=\operatorname{diag}(-1,1, \ldots, 1)$. It can be shown that the metric (29) is invariant with respect to a total Legendre transformation which changes the coordinates $\left\{\Phi, E^{a}, I^{a}\right\}$ to the coordinates $\left\{\tilde{\Phi}, \tilde{E}^{a}, \tilde{I^{a}}\right\}$ using the following algebraic rules,

$$
\Phi=\tilde{\Phi}-\tilde{E}_{a} \tilde{I}^{a}, \quad E^{a}=-\tilde{I}^{a}, \quad I^{a}=\tilde{E}^{a} .
$$

Applying the pullback $\varphi^{*}$ to the metric (29), we obtain the corresponding thermodynamic metric $g$,

$$
g^{G T D}=\varphi^{*}(G)=\left(E^{c} \frac{\partial \Phi}{\partial E^{c}}\right)\left(\eta_{a b} \delta^{b c} \frac{\partial^{2} \Phi}{\partial E^{c} \partial E^{d}} d E^{a} d E^{d}\right)
$$

which depends only of the fundamental potential $\Phi=\Phi\left(E^{a}\right)$. If we know the fundamental potential of the thermodynamic system that we want to study, the corresponding metric $g$ can be computed explicitly and the relations between thermodynamic and geometry can be also studied. 


\section{GEOMETROTHERMODYNAMICS IN MASSIVE GRAVITY}

Let us consider a thermodynamic system with two degrees of freedom. If we choose thermodynamic potential as $\Phi=M$ and the coordinates of equilibrium manifold as $E^{a}=$ $\{S, q\}$, then the corresponding metric is given as

$$
g^{G T D}=\left(S \frac{\partial M}{\partial S}+q \frac{\partial M}{\partial q}\right)\left(-\frac{\partial^{2} M}{\partial S^{2}} d S^{2}+\frac{\partial^{2} M}{\partial q^{2}} d q^{2}\right) .
$$

Using the expressions for the thermodynamic $M$, as given in Eq. (10), we obtain explicitly the GTD metric coefficients, which can be written as,

$$
\begin{aligned}
& g_{S S}^{G T D}=\frac{1}{32 \pi^{2}} \frac{\left[\left(\frac{S}{\pi}\right)^{\frac{\lambda}{2}}-q^{\frac{\lambda}{2}}\right]\left[\left(\frac{S}{\pi}\right)^{\frac{\lambda}{2}}-q^{\frac{\lambda}{2}}\left(1-\lambda^{2}\right)\right]}{\left(\frac{S}{\pi}\right)^{\lambda+1}}, \\
& g_{q q}^{G T D}=-\frac{1}{32}\left(\frac{S}{\pi}\right)^{1-\lambda} q^{\frac{\lambda}{2}-2}\left[\left(\frac{S}{\pi}\right)^{\frac{\lambda}{2}}-q^{\frac{\lambda}{2}}\right] \lambda(\lambda-2),
\end{aligned}
$$

and Eq. (32) takes the form

$$
g^{G T D}=\frac{1}{32 \pi^{2}} \frac{\left[\left(\frac{S}{\pi}\right)^{\frac{\lambda}{2}}-q^{\frac{\lambda}{2}}\right]}{\left(\frac{S}{\pi}\right)^{\lambda+1}}\left\{\left[\left(\frac{S}{\pi}\right)^{\frac{\lambda}{2}}+q^{\frac{\lambda}{2}}\left(\lambda^{2}-1\right)\right] d S^{2}-\lambda(\lambda-2)\left(\frac{S}{q}\right)^{2} q^{\frac{\lambda}{2}} d q^{2}\right\} .
$$

The curvature scalar corresponding to the metric (32) takes the form,

$$
R^{G T D}=-\frac{8 \pi \lambda\left(\frac{S}{\pi}\right)^{\frac{3 \lambda}{2}}\left[q^{\frac{\lambda}{2}}\left(2 \lambda^{2}+2 \lambda-3\right)+\left(\frac{S}{\pi}\right)^{\frac{\lambda}{2}}(3-2 \lambda)\right]\left[\left(\frac{S}{\pi}\right)^{\frac{\lambda}{2}}+(\lambda-1) q^{\frac{\lambda}{2}}\right]}{S(\lambda-2)\left[\left(\frac{S}{\pi}\right)^{\frac{\lambda}{2}}-q^{\frac{\lambda}{2}}\left(1-\lambda^{2}\right)\right]^{2}\left[q^{\frac{\lambda}{2}}-\left(\frac{S}{\pi}\right)^{\frac{\lambda}{2}}\right]^{3}}
$$

There are two curvature singularities in this case. The first one occurs if $q^{\frac{\lambda}{2}}-\left(\frac{S}{\pi}\right)^{\frac{\lambda}{2}}=0$ and corresponds to $M=0$, as follows from Eq.(10), see also figure 3. This means that this singularity is non physical since no black hole is present in this case. A second singularity is located at the roots of the equation $\left(\frac{S}{\pi}\right)^{\frac{\lambda}{2}}-q^{\frac{\lambda}{2}}\left(1-\lambda^{2}\right)=0$. For the interval $1-\lambda^{2}>0$, according to (21), it coincides with the points where $C \longrightarrow \infty$, i. e., with the points where second order phase transitions take place. The general behavior of the curvature scalar is illustrated in figures 4 . 

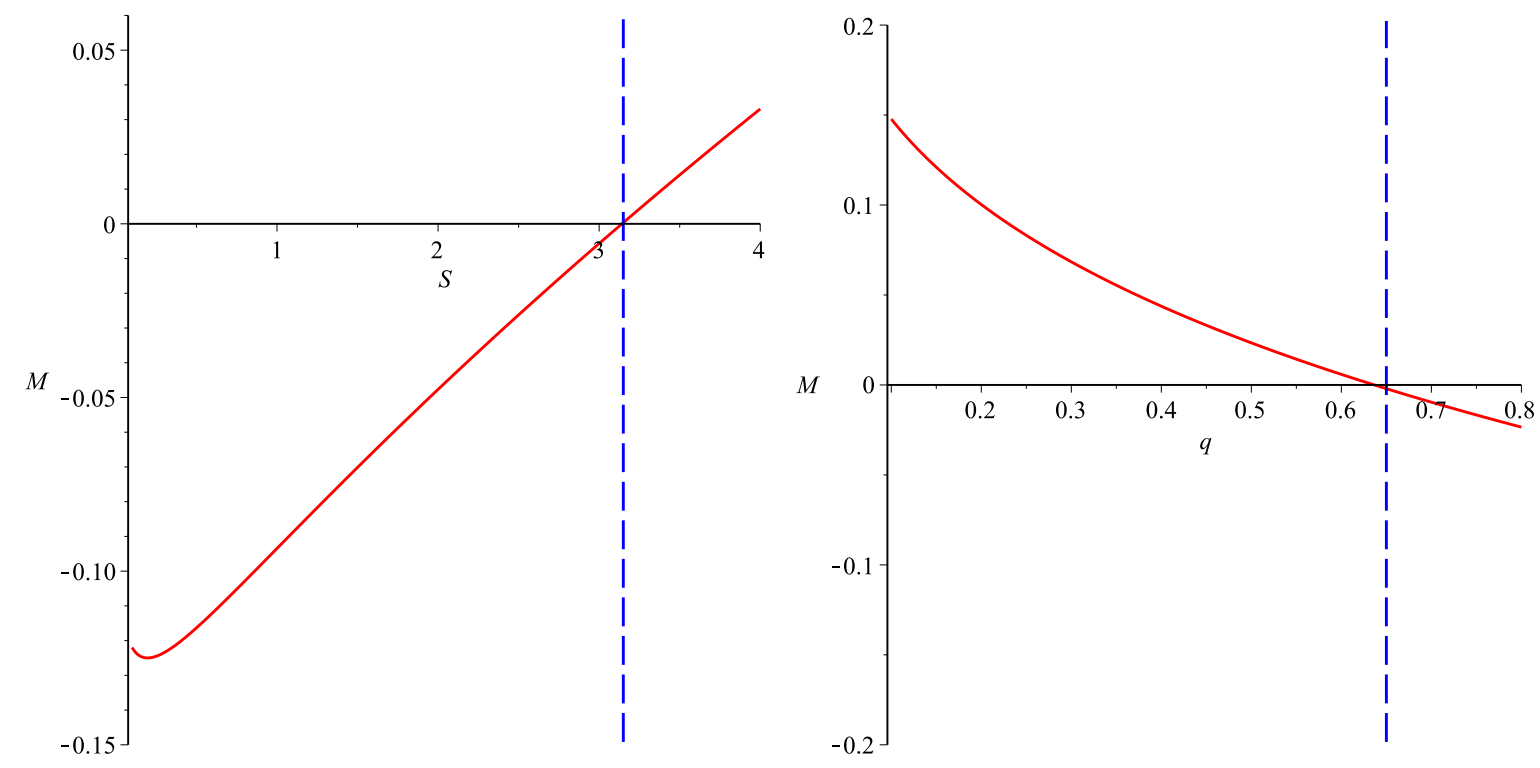

FIG. 3: The mass $M$ as a function of the entropy $S$ (left) and as a function of charge $q$ right, with $q=1$ and $S=2$, respectively. We have considered $\lambda=\frac{1}{2}$ in both cases.
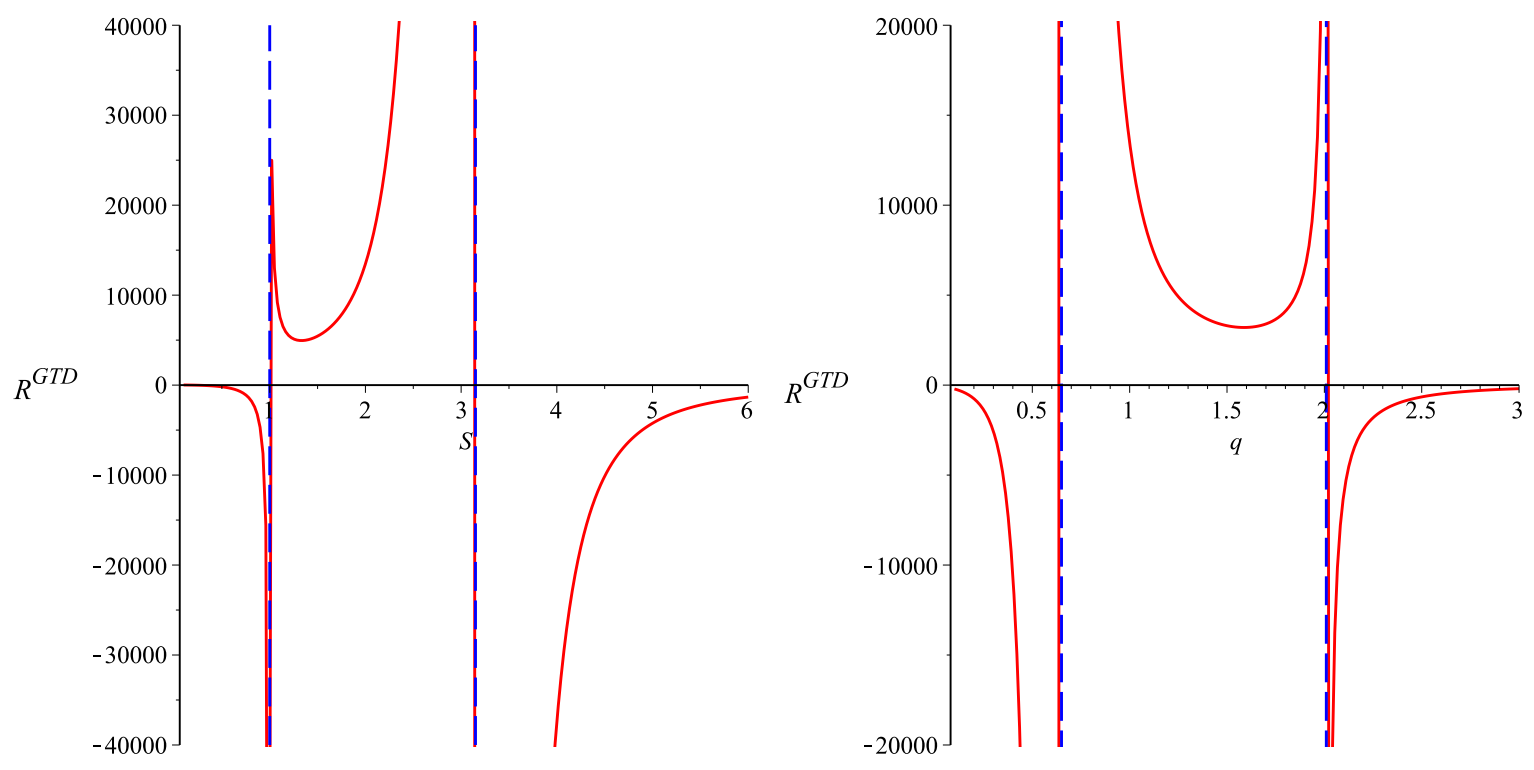

FIG. 4: The curvature scalar $R^{G T D}$ as a function of the entropy (left), with $q=1$, and as a function of charge $q$ (right), with $S=2$. We have considered $\lambda=\frac{1}{2}$ in both cases.

According to GTD, these results show that there exist curvature singularities at those points where second order phase transitions occur, because the denominators of the heat capacity and the curvature scalar coincide [27]. We can also observe, figure 4, that the 
curvature scalar changes spontaneously its sign indicating a transition of the a stable region to the unstable one, reproducing the thermodynamic behavior of this black hole.

\section{WEINHOLD AND RUPPEINER APPROACHES}

In this section we analyze the thermodynamic geometry of the same black hole in massive gravity by using Weinhold and Ruppeiner metrics.

The Weinhold metric is defined as [13]

$$
g^{W}=\left(\frac{\partial^{2} M}{\partial S^{2}} d S^{2}+2 \frac{\partial^{2} M}{\partial S \partial q} d S^{2}+\frac{\partial^{2} M}{\partial q^{2}} d q^{2}\right) .
$$

Using the fundamental thermodynamic equation $M=M(S, q)$, the metric (37) can be calculated and we obtain explicitly the Weinhold metric coefficients which can be written as,

$$
\begin{aligned}
& g^{W}=-\frac{1}{8 \pi^{2}\left(\frac{S}{\pi}\right)^{\frac{3}{2}(\lambda+3)}}\left\{\quad\left[\left(\frac{S}{\pi}\right)^{\frac{\lambda}{2}}+q^{\frac{\lambda}{2}}\left(\lambda^{2}-1\right)\right] d S^{2}-S q^{\frac{\lambda}{2}-1} \lambda(\lambda-1) d S d q+\right. \\
& \left.+S^{2} q^{\frac{\lambda}{2}-2} \lambda(\lambda-2) d q^{2}\right\}
\end{aligned}
$$

The curvature scalar corresponding to the metric (38) takes the form,

$$
R^{W}=0
$$

This result tells us that the space of thermodynamic equilibrium is flat, indicating the lack of thermodynamic interaction. Obviously, this result contradicts the results obtained by using black hole thermodynamics.

The case of Ruppeiner's geometry for thermodynamical systems must be computed in the entropy representation. However, as has been shown in [29] one can prove that Ruppeiner's metric, $g^{R}$, is proportional to Weinhold's metric, $g^{W}$ as $g^{R}=(1 / T) g^{W}$, where $T$ is the temperature. Using this result Ruppeiner's metric takes the form, 


$$
\begin{aligned}
& g^{R}=-\frac{1}{2 S\left[q^{\frac{\lambda}{2}}(\lambda-1)+\left(\frac{S}{\pi}\right)^{\frac{\lambda}{2}}\right]}\left\{\quad\left[\left(\frac{S}{\pi}\right)^{\frac{\lambda}{2}}+q^{\frac{\lambda}{2}}\left(\lambda^{2}-1\right)\right] d S^{2}-S q^{\frac{\lambda}{2}-1} \lambda(\lambda-1) d S d q+\right. \\
& \left.+S^{2} q^{\frac{\lambda}{2}-2} \lambda(\lambda-2) d q^{2}\right\}
\end{aligned}
$$

The corresponding curvature scalar can be written as

$$
R^{R u p}=\frac{\lambda\left(\frac{S}{\pi}\right)^{\frac{\lambda}{2}}\left[q^{\frac{\lambda}{2}}\left(\lambda^{2}+\lambda-2\right)-\left(\frac{S}{\pi}\right)^{\frac{\lambda}{2}}(\lambda-2)\right]}{S\left[\left(\frac{S}{\pi}\right)^{\frac{\lambda}{2}}+q^{\frac{\lambda}{2}}(\lambda-1)\right]\left[2 q^{\frac{\lambda}{2}}(\lambda-1)-\left(\frac{S}{\pi}\right)^{\frac{\lambda}{2}}(\lambda-2)\right]},
$$

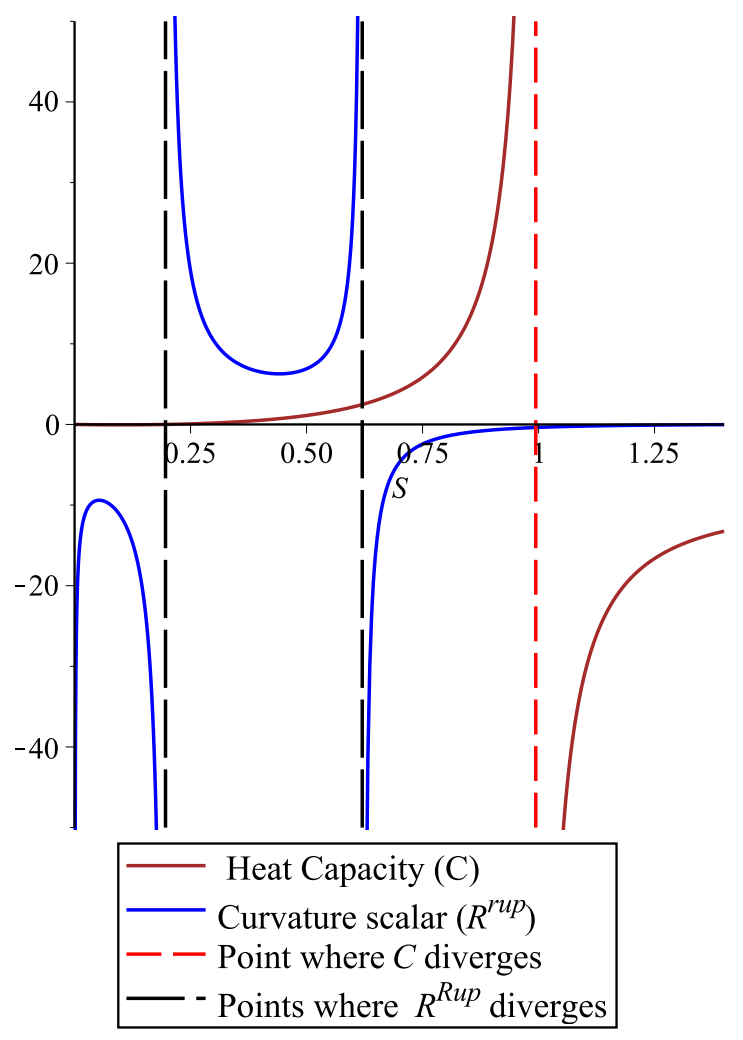

FIG. 5: The curvature scalar $R^{R u p}$ (in blue) and heat capacity (in brown) as functions of $S$, with $q=1$ and $\lambda=\frac{1}{2}$. The red dash thick vertical line corresponds to the point where the heat capacity is singular and the black longdash vertical lines represent the points where the curvature scalar of the Ruppeiner metric diverges. 
We can see that the Ruppeiner's geometry is curved, signaling interaction for this thermodynamic system. There are singular points, but they are not consistent with the divergencies of the heat capacity for fixed charge.

The fact that Ruppeiner's curvature scalar in this case doesn't diverge at the same points where the heat capacity does, tell us that it is not possible to associate curvature singularities with second-order phase transitions.

\section{CONCLUSIONS}

In this work, we analyzed the thermodynamic and geometrothermodynamic properties of a black hole solution in massive gravity. Using the fundamental equation of a spherically symmetric black hole we found the geometric properties of the corresponding manifold of equilibrium states. We found that the corresponding thermodynamic curvature turned out to be nonzero, indicating the presence of thermodynamic interaction. A numerical and analytical study of the thermodynamic curvature shows that the phase transitions which are characterized by divergencies of the heat capacity are described in GTD by curvature singularities in the equilibrium manifold.

We assumed in this work Davies's proposal to solve the problem of the lack of homogeneity of the fundamental equation. We have considered the definition (11), with which the fundamental equation (9) becomes a homogeneous function of degree $\frac{1}{2}$ in the extensive variables. We have also shown that for values of the constant $\lambda$ in the interval $\lambda^{2}-1<0$, the black holes in massive gravity presents a phase transition. Therefore, it would be interesting to investigate further $\lambda$ as a thermodynamic variable in order to learn more about the thermodynamics and phase transition structure of these black holes.

We analyzed the thermodynamic geometry based on the Weinhold metric and found that it represents a flat space, indicating the lack of thermodynamic interaction. We also analyzed the Ruppeiner's metric for this system and found that corresponds to a curved manifold for this black hole and the corresponding curvature diverges at some points, but these points are not the ones at where the heat capacity for fixed charge diverges. We conclude that the Weinhold and Ruppeiner's geometries do not describe correctly the thermodynamic geometry of black holes in massive gravity. We interpret this result as indication that it is necessary to take into account Legendre invariance in order to correctly describe 
thermodynamics from a geometric point of view.

\section{Acknowledgements}

This work was supported by Conacyt-Mexico, Grant No. 166391 and DGAPA-UNAM, Grant No.113514. I am grateful to Profs. F. Nettel and C. Lopez-Monsalvo for interesting comments, encouragement and support.

[1] J. F. Donoghue, Introduction to the Effective Field Theory Description of Gravity, arXiv:gr-qc/9512024

[2] E. J. Copeland, M. Sami and S. Tsujikawa, Int. J. Mod. Phys. D 15, 1753-1936 (2006).

[3] N. Arkani-Hamed, H.Georgi and M.D. Schwartz, Annals Phys., 305, 96-118 (2003).

[4] N. Arkani-Hamed, H.C Cheng, M.A. Luty and S. Mukohyama, JHEP,0405, 074 (2004) .

[5] S.L. Dubovsky, JHEP, 0410, 076 (2004).

[6] D. Comelli, F. Nesti and L. Pilo, Phys. Rev. D 83, 084042(2011).

[7] A. Bravetti, D. Momeni, R. Myrzakulov and H. Quevedo, Gen. Rel. Grav. 45,1603-1617 (2013).

[8] J. E. Åman, I. Bengtsson, and N. Pidokrajt, Gen. Rel. Grav. 35, 1733 (2003).

[9] J. E. Åman and N. Pidokrajt, Phys. Rev. D 73, 024017 (2006).

[10] J. E. Åman and N. Pidokrajt, Gen. Rel. Grav. 38, 1305 (2006).

[11] J. Shen, R. G. Cai, B. Wang, and R. K. Su, Int. J. Mod. Phys. A 22, 11-27 (2007).

[12] R. G. Cai and J. H. Cho, Phys. Rev. D 60, 067502 (1999); T. Sarkar, G. Sengupta, and B. N. Tiwari, J. High Energy Phys. 0611015 (2006).

[13] F. Weinhold, J. Chem. Phys. 63,2488 (1975).

[14] G. Ruppeiner, Phys. Rev. A 20, 1608 (1979).

[15] H. Quevedo, J. Math. Phys. 48, 013506 (2007).

[16] D. Blas, Aspects of Infrared Modifications of Gravity, arXiv:0809.3744v1 [hep-th].

[17] V. A. Rubakov,Lorentz-violating graviton masses: getting around ghosts, low strong coupling scale and VDVZ discontinuity, hep-th/0407104, (2004).

[18] D. Blas, D. Commelli, F. Nesti and L. Pilo, Phys. Rev. D, 80, 044025 (2009). 
[19] Capela F. and Nardini G, Phys.Rev. D 86, 024030 (2012).

[20] S.L. Dubovsky, P. Tinyakov and M. Zaldarriaga, JHEP, 0711, 083 (2007).

[21] M. V. Bebronne and P. G. Tinyakov, JHEP 0904, 100 (2009) [Erratum-ibid. 1106 (2011) 018].

[22] Z. Berezhiani, D. Commelli, F. Nesti and L. Pilo, JHEP 0807, 130 (2008).

[23] M. V. Bebronne, Theoretical and phenomenological aspects of theories with massive gravitons, arXiv:0910.4066 [gr-qc].

[24] P.C.W Davies,Proc. Roy. Soc. Lond. A, 353499 (1977).

[25] Mustapha Azreg-Ainou, European Physical Journal C 74, 1-8 (2014).

[26] H. Quevedo, Gen. Rel. Grav. 40, 971 (2008).

[27] H. Quevedo, A. Sánchez, S. Taj, and A. Vázquez, Gen.Rel.Grav. 43, 1153-1165 (2011)..

[28] G. Ruppeiner, Rev. Mod. Phys. 67, 605 (1995); 68, 313 (1996).

[29] R. Mrugala, Rep. Math. Phys. 21 197-203(1985).

[30] J. L. Álvarez, H. Quevedo, and A. Sánchez, Phys. Rev. D 77, 084004 (2008).

[31] A. Vázquez, H. Quevedo, and A. Sánchez, J. Geom. Phys. 60, 1942 (2010).

[32] H. Quevedo, A. Sánchez and A. Vázquez, Gen.Rel.Grav. 47 4, 36 (2015).

[33] H. Quevedo and A. Sánchez, JHEP 09, 034 (2008).

[34] H. Quevedo and A. Sánchez, Phys. Rev. D 79, 024012 (2009).

[35] H. Quevedo and A. Sánchez, Phys. Rev. D. 79, 087504 (2009).

[36] S. Weinberg. The cosmological constant problems, arXiv:astro-ph/0005265v1.

[37] S. Weinberg, Rev. Mod. Phys. 61, 1-23 (1989).

[38] G. Ruppeiner, Rev. Mod. Phys. 67, 605 (1995); 68, 313 (1996).

[39] A. J. M. Medved, Mod. Phys. Lett. A 23, 2149 (2008). 